\title{
Screening of different fibers for their prebiotic activity and potential systemic health benefit
}

\author{
A. Meynier, I. Moreira de Almeida, M. Tabernero-Urbieta and S. Vinoy \\ Kraft Foods Europe R\&D, 6 rue René Razel - Bâtiment K, 91400 Saclay, France
}

Prebiotic compounds are relevant candidates to improve health of the host as stated in the definition proposed by Gibson et al. (2004). Recent literature has implicated fermentable fibers in several health outcomes such as inflammation, carbohydrate metabolism or blood lipid composition. These outcomes are directly linked to metabolic syndrome which concern 20 to $25 \%$ of the world adult population according to the IDF. The aim of the present study was to evaluate the effect oftwo cereal and fruit fiberson gut microbiota composition and the production of SCFA. Inulin and cellulose were also tested as positive and negative controls, respectively. For this purpose, we used an in vitro model simulating the different parts of the digestive tract (TwinShime). Wheat arabinoxylans (AX) and citrus fibers (CF) were tested alone or in combination (50/50) at a daily dose of $5 \mathrm{~g}$ like inulin and cellulose. AX led to an increase in bifidobacteria and a decrease in lactobacilli populations. CF induced a small increase in bifidobacteria and lactobacilli (around $1 \log$ ) which was in the same range as the effect observed with inulin but this effect seems to appear later compared to inulin in the test. When $\mathrm{AX}$ and $\mathrm{CF}$ are combined (50/50) a small increase in bifidobacteria is observed $(+1 \mathrm{log})$ whereas great increase in lactobacilli population was measured ( $+2 \operatorname{logs}$ ), much higher than each ingredient alone. As for CF alone, the fermentation appears to be delayed in time and is still present during the wash-out period. As expected, cellulose induced a small decrease in both bifidobacteria and lactobacilli.In all cases except cellulose, a great increase in total SCFA production was quantified. AX as inulin tended to increase mainly butyrate whereas CF led mainly to a higher production of acetate. Combining AX and CF produced a large increase in acetate release.Moreover, for both $\mathrm{CF}$ and $\mathrm{AX}+\mathrm{CF}$ the SCFA production seem to confirm the delayed fermentation still present in the distal parts of the colon. Acetatehas been implicated by several authors in systemic beneficial effects such as modulation of glucose homeostasis, increase in GLP-1 and decrease in Ghrelin.

The combination of CF and AX seems to improve microbiota composition and may have systemic health benefits related to metabolic syndrome through the increased secretion of SCFA, especially acetate. These data obtained in vitro need to be confirmed in human. 